\title{
CAREGIVERSPRO-MMD: Community Services, Helping Patients with Dementia and Caregivers Connect with Others for Evaluation, Support and to Improve the Care Experience
}

\author{
Cristian Barrué ${ }^{1}$, Atia Cortés ${ }^{1}$, Ulises Cortés ${ }^{1,2}$, Frédéric Tétard ${ }^{3}$, Xavier Gironès ${ }^{4}$ \\ ${ }^{1}$ Universitat Politècnica de Catalunya, Computer Science Department, Barcelona, \\ Spain \\ ${ }^{2}$ Barcelona Supercomputing Center, Barcelona, \\ Spain \\ ${ }^{3}$ Mobiles Dynamics, Barcelona, \\ Spain \\ ${ }^{4}$ University of Vic - Central University of Catalonia, Faculty of Health Sciences, Manresa, \\ Spain
}

cbarrue@cs.upc.edu, acortes@cs.upc.edu, ia@cs.upc.edu, ftetard@mobilesdynamics.com, xgirones@umanresa.cat

\begin{abstract}
In this paper, we present the design of a mHealth application that is specifically targeted to caregivers and persons with mild to moderate dementia. The result is CAREGIVERSPRO-MMD: a tool integrating a broader diagnostic approach, incorporating the live-in family caregiver-patient dyad and considering this dyad as the unit of care. CAREGIVERSPRO-MMD will provide value-added services based on social networks, tailored interventions, clinical strategies and gamification for improving the quality of life for people living with dementia and their caregivers, allowing them to live in the community for as long as possible. CAREGIVERSPRO-MMD is an EU project funded under the $\mathrm{H} 2020$ program.
\end{abstract}

Keywords. mHealth, active and assisted living, mild cognitive impairment, caregiver, dementia, social network.

\section{Introduction}

According to the World Health Organisation [31], $47 \mathrm{M}$ people around the world have some form of dementia, for which there is no effective intervention, to halt or reverse the progressive cognitive impairment. As Europe's population is ageing, long-term care for elderly citizens will become an increasing cost for society. To manage this transition healthcare policies in the EU and individual Member States are heavily focused on extending the independent life of the elderly, with the dual aim of increasing their quality of life and reducing the costs of care.

Health delivery practices are shifting towards home care. The reasons are the better possibilities for managing chronic care, controlling health delivery costs, increasing quality of life and quality of health services and the distinct possibility of predicting and thus avoiding serious complications [14]. The concept of social networks using mobile devices to support health communities has emerged as a new and viable reality in the field of IT for health and telemedicine under the funding of the EU programs. The so-called $\mathrm{mHealth}$ has the "potential to transform the face of health service delivery across the globe", and 
there is increasing media and consumer interest in mHealth, illustrated for example by a recent panel at the US Consumer Electronics Show on The Digital Health Revolution [16, 25, 30].

The Ageing of Europe is characterised by a decrease in fertility, a decrease in mortality rate, and a higher life expectancy among European populations, together with continued, but decelerating inward net migration to the EU. As a result, the proportion of people of working age in the EU is shrinking while the relative number of those retired is expanding. Ageing is one of the greatest social and economic challenges of the $21^{\text {st }}$ century for World societies. It will affect all EU countries and most policy areas. In all Europe, life expectancy is increasing in an almost continuous and uniform trend at the rate of 2-3 months every year, and is the main driver behind the population ageing. By 2025 more than $20 \%$ of Europeans will be 65 or over, with a particularly rapid increase in numbers of over-80s. Because older people have different healthcare requirements, health systems will need to adapt so they can provide adequate care and remain financially sustainable.

In countries like Mexico in 2010 was not yet facing a health crisis related to demographic ageing and it was just at the right time to develop health care and prevention policies and programmes based on the research and experiences of developed countries to cope with the situation [22]. Still, this effort has to be done. As explained by Robledo et al, in 2012, nine percent of the Mexican population is over 60 years old, with an annual growth during the last decade of close to $4 \%$, in two decades or less, this segment of the population will reach $20 \mathrm{M}$ people; however, even with just half of that population, the country is not currently equipped to face this problem [23]. A recent study by the National Institute of Health and Ageing in Mexico (ENASEM) found that $70 \%$ of older adults do not receive the medical services they need in either public or private institutions. Nor do they have much money to pay for them: on average, it was reported, the typical senior in Mexico has a monthly income of around 2,000 pesos, or US 120 , and only $25 \%$ have a retirement or pension plan [10].
CAREGIVERSPRO-MMD is focused on people living with Mild Cognitive Impairment or Mild to Moderate Dementia (PLWD from now on) and their caregivers. Mild Cognitive Impairment $(\mathrm{MCl})$ is a condition that falls somewhere between normal age-related memory loss and Alzheimer's disease or a similar impairment. Not everyone with $\mathrm{MCl}$ develops dementia. And like dementia, $\mathrm{MCl}$ is not an illness, but a cluster of symptoms that describe changes in how you think or process information. Memory problems are the most common indicators of $\mathrm{MCl}$. A person with $\mathrm{MCl}$ may also experience difficulties with judgement, orientation, thinking and language beyond what one might expect with normal ageing. For unknown reasons, $\mathrm{MCl}$ appears to affect men more than women.

\subsection{The Old-age Dependency Ratio}

The ratio of working-age people to the people over 65 years of age is expected to drop in the EU from 4 to 2 . The increase in the total age-dependency ratio (people aged 14 and below and aged 65 and above over the population aged 15-64) is projected to be even larger, rising from $49.3 \%$ in 2010 to $77.9 \%$ in 2060 . This will directly reduce the growth rates of the European economies by slowing the growth of the capital stock and by weakening the productivity of the labour force. Thus, a smaller tax base and less tax revenues are expected. In addition, the current tax-financed systems of social pensions and healthcare will require substantial increases in the already high tax rates. Continued increases in longevity will ensure that the old-age dependency ratio, which measures the number of elderly people as a share of those of working age, will rise sharply in most countries over the next 40 years. Most of the rise in the old-age dependency ratio is expected by the early 2030s, that makes the next two decades as a time of transition in (i) public policies affecting the elderly like Social Security and Medicare; (ii) how we start building systems that can support families and communities in providing assistance and care for the elderly who need it.

Due to an ageing population, the public provision of long-term care poses an increasing challenge to the sustainability of public finances in the 
EU. The ageing of population is expected to put pressure on governments to provide long-term care services as old people often develop multi-morbidity conditions, which require long-term medical care and assistance with a number of daily tasks. Health trends among the elderly are mixed: severe disability is declining in some countries but increasing in others, while mild disability and chronic disease are generally increasing. This situation is comparable in many societies around the world.

The structure of this paper is the following. In $\S 2$ we will address related work explaining relevant mHealth platforms implemented to give support to elder individuals in their Activities of Daily Living. Section 3 will develop the CMMD architecture, deployment and its goals. In $\S 4$ we explain the objectives of the pilot study and give insight information about the CAREGIVERSPRO-MMD stakeholders. In $\S 5$ we will discuss our conclusions and comment the future work.

\section{Related Work}

It is well accepted that people living connected to their relatives or community-dwellers have a better quality of life that helps them maintaining a healthy life-style. People with few social relations are exposed to social exclusion and, thus, have a higher risk of mortality. This situation is more critical for those seniors having mobility impairment, as they tend to reduce both their social and physical activities, leading to a motor and cognitive decline. Usually, relatives are affected by this situation as they have to take care of their seniors, take them to medical visits and be constantly aware of what they are doing.

The relation among doctors, patients and relatives has been empowered during the last years by the emergence of specific social networks. These can be centred in a given stakeholder (the patient, the doctor or the caregiver), so the main services of the platform will focus on assisting them. Other social networks aim to include all stakeholders at a same level, improving the communication among them while monitoring a patient's activities of daily living. The evolution on mobile devices, bio-medical sensors and new forms of ubiquitous communication, such as social networks, are essential for the new generation of healthcare systems. The Internet of Things is expected to empower the potential of mobile health services in the near future, offering standards of communication among different entities and integrating different care monitoring and sensing systems.

Online social networking sites have become the major channel by which people develop their personal relations in recent years. They provide, by definition, a new method of communication, employing computers as a collaborative tool to accelerate group formation and to escalate group scope and influence. Social networks allow the individual to construct his/her profile providing data about himself, sharing text, images, and photos, and to link other members of the site by applications and groups provided on the Internet. Hence, social networks enable users to introduce themselves, connect to social circles, and develop and maintain relationships with others. Social networks are currently the world's fastest developing personal networking tool.

A social network is a type of online community of individuals who interact with one another to pursue common goals and/or interests. Online community sites are sometimes considered as social network sites, though in a broader sense, social network sites usually provide an individual-centred service whereas online community services are groupcentred, bounded together by a common interest or topic. Some examples of this new medical interaction are based on an online peer-to-peer support networks, such as PatientsLikeMe [20], MedHelp [15], PatientPower [18] or Crohnology [6], where patients can manage their own health status by uploading and monitoring their medical history. They can also contact other individuals who have similar diagnoses and compare them or share their personal experiences. These tools can improve the patient care and, in the end, their quality of life. Social networks already have demonstrated potential empowering Activities of Daily Living (ADL)for the elderly [2].

Some social networks aim to connect physicians in medical platforms where they can share experiences, publications, treatments or professional 
consultations. The most popular platforms are Doximity [8], Sermo [24] and QuantiaMD [21].

Other solutions, like PatientsKnowBest [19] or WebMD [29], seek for the communication between patients and doctors, allowing online consultations from home. This kind of platforms are expected to cause an economic impact for public and private health care systems, as well as a social impact for patients and relatives. People having mobility impairment and dependence will benefit of remote health monitoring and it will improve the medical attention efficiency.

Finally, there are several online communities focused on caregivers and/or patients where they will share experiences, advertise for interesting events or publications and support among them. Some of these platforms, like Voisinage [28], are used also as a collaborative tool, where both elderly and young people connect and try to help each other by reciprocally offering care and community services. These online communities are usually free web-based, inspired in the format of blogs with some internal chat for connecting people with similar conditions. Other examples are ConnectedLiving [5], CarePages [4] or Vincles [27] that is supported by the Barcelona City Council.

Konstantinidou et al. (2015) proposed a cloud-based architecture to support Active Assisted Living ( $A A L$ ) services enhanced by semantic ontologies of the existing scientific knowledge about people with $\mathrm{MCl}$ and their needs to lead to resource sharing under minimal management efforts.

BREATHE project [26] is funded by the the Active and Assisted Living Joint Program (AAL) and aims to provide an ICT solution to address and improve caregiving at the personal, local, and European levels. Despite BREATHE does not focus on dementia care, it plans to develop two very interesting public whitepapers, about The needs and requirements of $A A L$ and ICT solutions for informal long-term care of elderly people in order to identify those challenges that should be addressed in both a socio-economic as well as scientific-technological domains and $A A L$ systems and associated privacy issues describing how ethical issues can be applied in order to have a successful vision and sensors-based supporting systems for elderly people.

Social networks are not only expected to empower social interaction among elderly people, but also to offer a new range of information to doctors, caregivers and relatives. They are more likely to share personal experiences with persons that live in similar conditions and thus the experts may be able to forecast diagnosis, the state of the relation among patients and their environment, or the success of a given treatment or drug from the information extracted from the network. In this direction, CMMD aims to study the dyad relation among a network of caregivers and PLWD and at the same time offer them tailored support.

\section{CAREGIVERSPRO-MMD}

CAREGIVERSPRO-MMD (CMMD) is a EU funded project under the H2020 programme devoted to build a mHealth application that is specifically targeted to caregivers and people with cognitive impairment or mild to moderate dementia. CMMD is a tool integrating a broader diagnostic approach, incorporating the live-in family caregiver-people living with dementia (PLWD) dyad and considering this dyad as the unit of care.

The project comprises three phases: first, to design and develop the first prototype of the mHealth application considering previous work on dementia and psychiatric co-morbidity symptoms, screening and intervention strategies to be implemented by the platform.

In the second phase, a user-centric analysis will be conducted to re-design the existing prototype for PLWDs. The development will be steered by PLWDs, caregivers and doctors, through user-centric design: we will collect feedback on each new version of the application until the design is adapted to the users' conditions.

In the third phase, the optimised application will be piloted with 600 dyads (PLWDs and their respective caregivers) and 600 controls. This will show the clinical and social benefits for PLWDs and caregivers, as well as financial benefits for the healthcare system.

CMMD is an intelligent support platform promoting Quality of Life (QoL), well-being and medication 
compliance for PLWD and caregiver in the community at the point of care. It will be available through smartphones and tablet computer, web browser and SMS text messaging. Its interface is being designed to suit users with low IT familiarity [17]. Additionally, CMMD will be compliant with internal security protocols and policies as well as industry regulatory policies, it is designed to only collect and process data concerning health for specific and legitimate purposes.

The novelty of $C M M D$ consists into integrating a broader diagnostic approach, incorporating the live-in family dyad as the unit of care. Some of the needs that PLWD and their informal caregivers currently perceive as insufficiently met by regular care and support services might be alleviated, or even be met with the help of the mutual assistance communities, using CMMD. These needs can be summarized as (1) the need for general and personalized information; (2) the need for support with regard to symptoms of dementia; (3) the need for social contact and company; and (4) the need for health monitoring and perceived safety [13].

CMMD will learn from their experiences and evolution integrating different types and sources of data, personalized screening results, tailored educational contents, clinical guidelines as intervention plans, and knowledge-based decision support tools, always considering privacy and security issues.

CMMD will use social networks (SN) services for organising and coordinating care at the level of the individual, with the aim of providing long-term care for people with dementia in the community as an alternative to early admission to a care home or hospital. Using SN services, CMMD will create, promote, and support a family of secure online communities through five levels of interaction, describing the social network model (see figure 1):

C1 A public community to increase awareness and prevention on cognitive impairment symptoms and support. This includes awareness raising campaigns on PLWDs' needs and caregivers' needs and on available ICT-based tools and their benefits;
C2 A private secure online community to share experiences and tacit knowledge between caregivers on health management and to find support;

C3 A private secure online community to coordinate and manage care at the point of care in the community, equipped with a number of services that help the C3 community to manage the PLWD and care more effectively. C3's members are trusted people. Doctors and social caregivers can connect to this trusted and private community.

C4 A private secure online community to share experiences and tacit knowledge between patients on health management and to find support;

C5 A private secure online community devoted to the pure and genuine health dimension of cognitive impairment where General Practicioner, Specialist, Psychologist, etc, have access to the platform, accessing contents, evaluating data, providing their professional contents.

Patient-reported outcome measures will be used to evaluate disease and treatment progression in many therapeutic dimensions, capturing relevant aspects of the disorder. Figure 2 shows the CMMD's platform look \& feel for a caregiver user. The User interface will be health condition based. Using Social and Clinical screening service for people with $\mathrm{MCl}$ or mild to moderate dementia presents challenges given the presence of cognitive impairment and loss of insight [11].

One of the CMMD's main objectives is to detect health changes for PLWD and for caregivers. The screening service draws information from validated clinical check-lists of questions to identify dementia symptoms and psychiatric co-morbidity for PLWDs, symptoms of burnout and psychiatric co-morbidity for caregivers and well-being and QoL for both. The PLWDs administer their own evaluations if they are still able to interact with the platform or their caregivers help them to administer his/her evaluations otherwise.

Figure 3 shows the different categories of personal data required to complete a user 


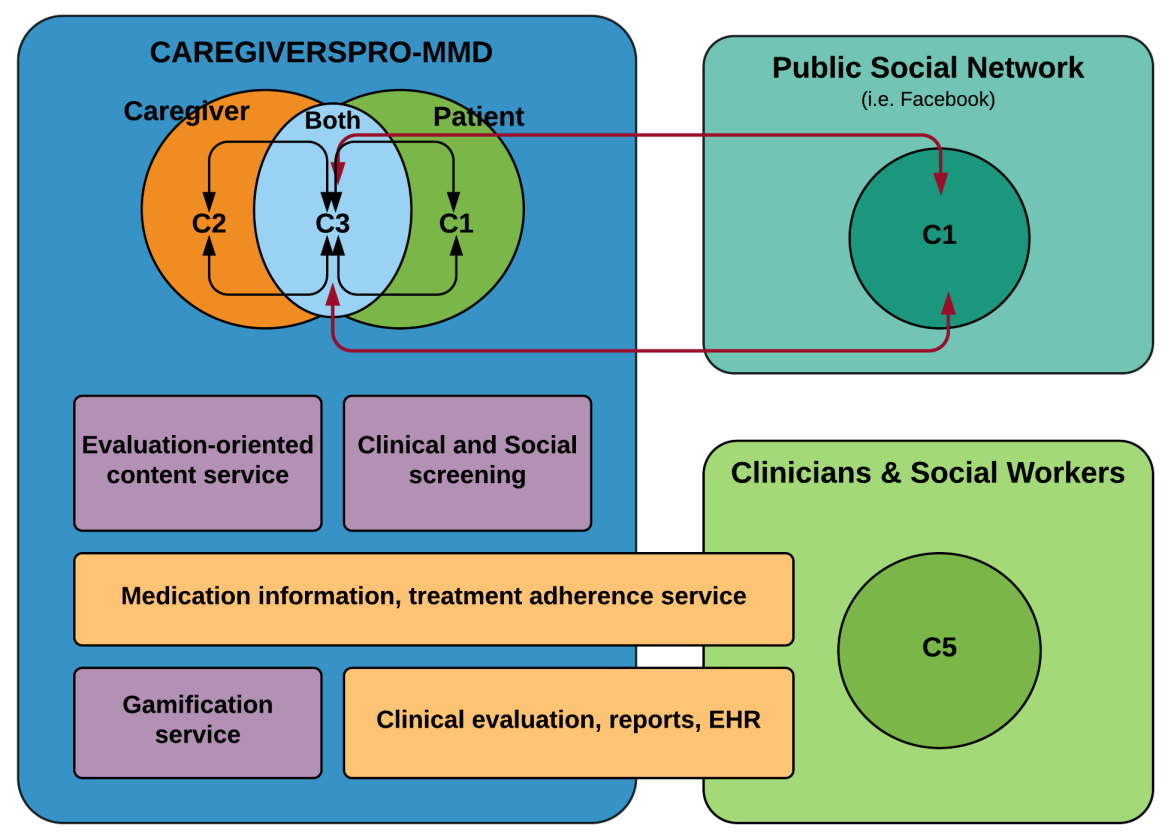

Fig. 1. $C M M D$ Architecture

profile. Any processing of personal data must be compatible with the CMMD purposes, as well as communicated to the users of the $C M M D$ platform. Secondary processing of anonymised data for scientific and historical research purposes or statistical purposes are considered to be compatible with the original purposes if they are done in accordance with any national or EU level rules adopted for it.

The therapeutic educational interventions is another CMMD's important service. This service offers personalized educational contents to their PLWDs and caregivers through the social network feed tailored to their specific profile. The educational focus is two-fold: first, it offers generic contents focused on dementia disease, dementia and psychiatric co-morbidity symptoms and available resources on the community and secondly, it proposes user-tailored content triggered on the basis of screening results to counter identified symptoms.

The CMMD platform regularly reminds PLWDs and caregivers of the need to take medication, helping them to comply with treatment and promoting adherence. Treatment adherence is another component of the platform in charge of reinforcing treatment compliance of the users, which barely reaches $50 \%$ in this kind of population [7]. Compliance level is going to be measured by: a) gamified strategies to gather adherence rates without burdening the user with questions; b) clinically validated treatment adherence questionnaire result from the CMMD screening service.

\subsection{CMMD Design Rationale}

The CMMD platform is built over low-coupling, reusable, modular components that implement services. The system architecture is as simple as possible to maintain yet meet all requirements. Whenever complexity is required, it must be encapsulated to promote simplicity of solutions built on the architecture. Reusable components represent opportunities to reduce IT development times and costs. Those components 


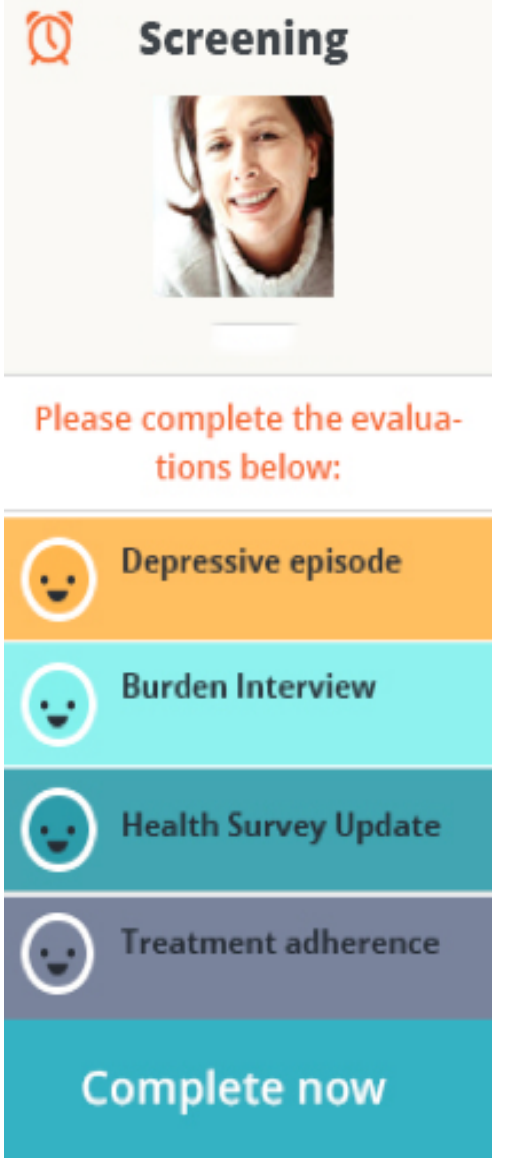

Fig. 2. $C M M D$ Screenshot

leverage investments in current systems. Modular components increase the systems' capacities to adapt to different evolution needs, because the change is easily isolated from affected modules.

Since tablets appear more attractive to elderly people and portability will play an important role in $C M M D$, the proposed framework will be inspired by the Mobile Cloud Computing (MCC) for use under various contexts (clinic, home, community) [1]. CMMD requires that the system selects the proper set of services based on the characteristics of the user, the user recent activity history, and psychological and cognitive health status. ideally this profiling phase is made by the doctor who will prescribe the solution and setup the initial caregivers and PLWD data. Then, semantic matchmaking will be applied: (i) to user profiling according to a user classification schema and (ii) on the sensing component according to screening scales' ontology (supported neuropsychological and behavioural validated scales).

\section{CMMD Study}

CMMD requires an assessment of four factors: (i) interest in and motivation to use the platform; (ii) ability to successfully use the technology; (iii) determination if the actual actors actually employ the platform as intended; and, where appropriate, (iv) whether actors continue to use the technology. This evaluation requires two steps: (i) a proof of concept demonstration that the CMMD platform can operate as intended; and (ii) a demonstration that targeted population (PLWD and caregiver, elderly people) can, want to, and will use the product as intended to achieve the ends as advertised [12]. To probe the second part we are preparing a large study including the deployment of the technology in four pilots.

\subsection{CMMD Hypotheses and Objectives}

The basic hypotheses of this study is that the use during 18 months of the CMMD platform has a benefit for the dyad, in the subjective QoL of persons living with $\mathrm{MCl}$ or dementia (mild to moderate dementia) and in the level of burden experienced by the primary caregiver. Also, it is expected that this use will be beneficial in their treatment adherence, behavioural and psychological symptoms and use of psychotropic drugs for both sides of the dyad. For this end there it will be four pilots, see Table 1. Pilots will assess individuals (patient and informal caregiver) on admission throughout a comprehensive geriatric assessment using standardized scales.

The main objectives are:

1. for PLWDs, to evaluate their subjective QoL in order to identify a benefit from the use of the CMMD platform during 18 months, and 


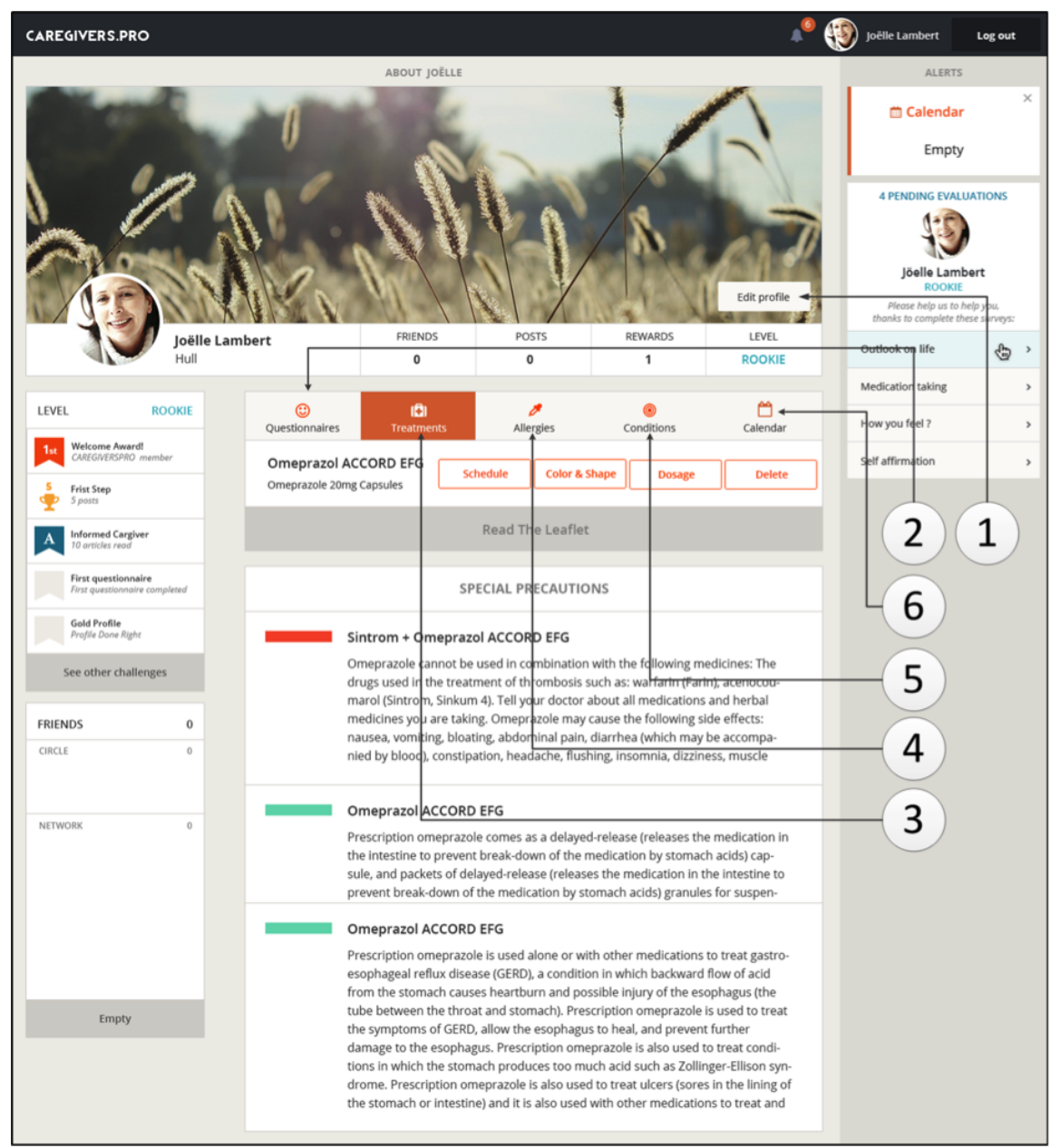

Fig. 3. User profile: (1) General information about the user (demographics, interests, health information, privacy settings); (2) Questionnaires; (3) Treatments (schedule, dosage, reminders); (4) Allergies (to prevent potential drug adverse effects); (5) Conditions (only editable by health professionals); (6) Calendar with events

2. for primary caregivers of persons living with $\mathrm{MCl}$ or dementia (mild to moderate dementia), to evaluate their perceived burden in order to identify a benefit from use of the $C M M D$ platform during 18 months.

Secondary objectives include the evaluation of:

1. the number of hospitalisations for PLWDs;

2. the perceived social support, success in relationships, self-esteem, purpose and optimism for caregivers, as well as the use of psychotropic drugs for these stakeholders;
3. the quality of the care-giving relationship between the caregiver and the PLWD;

4. the direct and indirect costs of care, and

5. the degree of satisfaction of using CAREGIVERSPRO-MMD.

We will be able to measure cultural differences due to the localization in four different countries and languages. 
Table 1. CAREGIVERSPRO-MMD will run 4 pilots. A total of 600 dyads (patient-caregiver) will be enrolled across Europe

\begin{tabular}{|l|c|c|}
\hline Country & \% Population $>65$ & Size Cohort \\
\hline France & $17 \%$ & 100 \\
\hline Italy & $21 \%$ & 200 \\
\hline Spain & $21 \%$ & 200 \\
\hline UK & $17 \%$ & 100 \\
\hline \hline
\end{tabular}

\subsection{CMMD Stakeholders}

- PLWD: People living with Mild Neurocognitive Disorders or Mild to Moderate Neurocognitive Disorders. Participants who belong in this category are divided into two subgroups depending on the severity of their symptoms according to the diagnosis made by their doctor.

- Caregivers: Formal and informal caregivers (no matter if they are paid or not) are people who provide care to PLWD individuals with his or her activities of daily living.

- Health Professionals: as health care professionals (doctors, nurses, psychologists, geriatrics, specialists, etc.) who work as primary care provider of a PLWD in a hospital, skilled nursing facility or clinic. Those people are expected to make diagnoses and set treatment plans for PLWD and caregivers.

- Social workers: are professionals who work with people with neurocognitive disorders and their families to provide support and help in order to improve outcomes in people's lives. They maintain professional relationships with people and their families may work for the state and are especially interested on the social status of PLWD.

- Administrator of the CMMD services.

\subsection{Previous to the Platform Deployment}

The CMMD consortium is preparing the deployment of the CMMD platform. A PACT analysis [3] has been performed considering feedback from the main stakeholders in focus groups to gather requirements on the design and usability of the current CMMD platform and to explore barriers and facilitators to using and engaging with the platform to help with the design of the pilot study. Results were already presented in [9]; following is a summary of those results:

- Platform design: Users reported that bigger font size and more contrast in colour between the font size and the background could help them engage with the platform. Being able to enlarge the screen or the text would also be helpful. Professionals reported that a simple and clear interface, together with audio instructions, would help older people use the platform.

- Content: PLWD reported no interest in games and online questionnaires, and do not think medication reminders would be helpful. On the other hand, caregivers believe that online questionnaires about memory and mood, as well as brain training games may be beneficial for PLWD. In addition, professionals suggested using short generic questionnaires to self-monitor health and well-being. Similarly, caregivers and professionals believe that medication reminders can be helpful. Finally, all three user groups believe that PLWD and caregivers may benefit from the social network function of the platform as well as information on dementia, caregiver support, healthcare advice and available groups and activities in the local area.

- Sharing Information: All user groups reported concerns in the security for sharing personal information online.

- Barriers: For PLWD: lack of knowledge in using ICT/technology; not having access to Internet; lower ability to learn new information. For caregivers and professionals: lack of time and security concerns regarding personal information.

- Facilitators: Simple, uncluttered interface designs; using touchscreen tablets; supporting PLWD in using the platform. 


\section{Conclusions and Future Work}

Elderly people with cognitive impairment feel there is a great need for more public awareness of the disease and more support for caregivers. While treatments to reverse or halt disease progression are not available for most of the dementias, and for the foreseeable future, treatment and medication of dementia will remain centred entirely on disease management. Slowing down the rate of cognitive decline and early diagnosis is essential in this as this gives PLWDs the best chance to maintain their cognitive ability. It also allows caregivers and PLWDs to plan for the future. CMMD supports PLWDs and caregivers, providing a useful screening tool to identify dementia symptoms, psychiatric co-morbidity and tailored interventions at the point of care in the community.

Results of studies like the one discussed in $\S 4.3$ brought in-deep information useful to adapt and up-grade. The design and content of the CMMD platform will be updated with further focus groups in France, Italy and Catalonia, interviews and feasibility studies will be conducted to test the updated versions of the platform [9].

\subsection{Future Work}

CMMD will use Gamification strategies as an innovative approach to enhance end-user motivation to utilize the platform. The design of an incentive scheme for CMMD's end-users, including doctors and social carers, is a major algorithmic challenge, since such a rewarding model must be fair, credible and respect the user's privacy, promoting their emotional self-integration and social acceptance and inclusion.

\section{Acknowledgements}

Authors are partially supported by the project Self-management interventions and mutual assistance community services, helping patients with dementia and caregivers connect with others for evaluation, support and inspiration to improve the care experience (CMMD) H2020-PHC-2015 \#690211. The views expressed in this paper are not necessarily those of the consortium CMMD.
Prof. Cortés is a member of Sistema Nacional de Investigadores (SNI-III), (CONACyT, Mexico).

\section{References}

1. Aloulou, H., Mokhtari, M., Tiberghien, T., Biswas, J., \& Yap, P. (2014). An adaptable and flexible framework for assistive living of cognitively impaired people. IEEE journal of biomedical and health informatics, Vol. 18, No. 1, pp. 353-360.

2. Barrué, C., Cortés, A., Moreno, J., PérezPasalodos, M., \& Cortés, U. (2015). Using multi-agent systems to mediate in an assistive social network for elder population. Proc. of the $18^{\text {th }}$ Int. Conf. of the Catalan Association for Artificial Intelligence, pp. 120-129.

3. Benyon, D. (2005). Designing Interactive Systems: People, Activities, Contexts, Technologies. Addison Wesley.

4. CarePages (2015). https://www.carepages.com/.

5. ConnectedLiving http://www.connectedliving.com.

(2015).

6. Cronhnology (2015). http://www.cronhnology.com.

7. DiMatteo, M. (2004). Variations in patients' adherence to medical recommendations: a quantitative review of 50 years of research. Medical Care, Vol. 42, No. 3, pp. 200-209.

8. Doximity (2015). https://www.doximity.com.

9. Dunn, R., Zafeiridi, P., Wolverson, E., \& Paulson, K. (2016). Consulting end-users in the design and usability of CAREGIVERSPRO-MMD: An internet based support tool designed for people with dementia and their caregivers. Alzhiemer Europa, pp. PO3.29.

10. ENASEM (2013). Estudio Nacional de Salud y Envejecimiento en México. http://www.inegi.org.mx/est/contenidos/proyectos /encuestas/hogares/especiales/enasem/doc lenasem2013_09.pdf.

11. Frank, L., Lenderking, W. R., Howard, K., \& Cantillon, M. (2011). Patient self-report for evaluating mild cognitive impairment and prodromal Alzheimer's disease. Alzheimer's research \& therapy, Vol. 3, No. 6, pp. 1.

12. Kearns, W. \& Fozard, J. (2016). Evaluating new gerontechnologies: Proof of concept is necessary, but not sufficient. Gerontechnology, Vol. 14, No. 3, pp. 139-145. 
13. Lauriks, S., Reinersmann, A., Van der Roest, H. G., Meiland, F., Davies, R. J., Moelaert, F., Mulvenna, M. D., Nugent, C. D., \& Dröes, R.-M. (2007). Review of ICT-based services for identified unmet needs in people with dementia. Ageing Research Reviews, Vol. 6, No. 3, pp. 223-246.

14. Maglaveras, K. V., Chouvarda, I., Goulis, D., Avramides, A., Adamidis, D., Louridas, G., \& Balas, E. (2002). Home care delivery through the mobile telecommunications platform: the Citizen Health System (CHS) perspective. Int J Med Inform., Vol. 68, No. 1-3, pp. 99-111.

15. MedHelp (2015). http://www.medhelp.org/.

16. Muuraiskangas, S., Harjumaa, M., K., K., \& Ermes, M. (2016). Process and effects evaluation of a Digital Mental Health Intervention targeted at improving occupational well-being: Lessons from an intervention study with failed adoption. JMIR Ment Health, Vol. 3, No. 2.

17. OECD (2013). Skills Outlook 2013: First results from the survey of adult skills. https://www.oecd.org/skills/piaac/Skills.

18. PatientPower http://www.patientpower.info/.

(2015).

19. PatientsKnowBest (2015). http://www.patientsknowbest.com.

20. PatientsLikeMe https://www.patientslikeme.com/.

21. QuantiaMD (2015). http://www.quantiamd.com.
22. Rico-Verdín, B., Rodriguez-Abrego, G., Villaseñor-Ruiz, I., \& Torres-Cosme, J. (2010). Burden of disease in elderly mexican population. In Handbook of Disease Burdens and Quality of Life Measures. Springer, pp. 763-782.

23. Robledo, L. M. G., Ortega, M. L., \& Lopera, V. E. A. (2012). The state of elder care in mexico. Current Translational Geriatrics and Experimental Gerontology Reports, Vol. 1, No. 4, pp. 183-189.

24. SERMO (2015). http://www.sermo.com.

25. The PLOS Medicine Editors (2013). A reality checkpoint for mobile health: Three challenges to overcome. PLoS Medicine, Vol. 10, No. 2:e1001395.

26. BREATHE (2015). http://www.breathe-project.eu.

27. VINCLES BCN (2015). The vincles barcelona care net. http://mayorschallenge. bloomberg.org/ideas/collaborative-care-networksfor-better-aging/.

28. Voisin-Age (2015). http://www.voisin-age.fr.

29. WebMD (2015). http://www.webmd.com.

30. WHO (2013). Call for innovative health technologies. http://www.who.int/ehealth/en/.

31. WHO (2016). Dementia fact sheet. http://www.who.int/mediacentre/ factsheets/fs362/en/.

Article received on 28/11/2016; accepted on 12/01/2017. Corresponding author is Cristian Barrué. 